\title{
Multiple Myeloma
}

\section{Abstract 1995}

\begin{abstract}
Subcutaneous Daratumumab in Patients With Relapsed or Refractory Multiple Myeloma: Part 2 Safety and Efficacy Update of the Open-Label, Multicenter, Phase 1b Study (PAVO)

Ajai Chari, MD, Maria-Victoria Mateos, MD, PhD, Niels W. C. J. van de Donk, Jonathan L. Kaufman, MD, Philippe Moreau, Albert Oriol, MD, Torben Plesner, MD, DSc, Lotfi Benboubker, MD, Hareth Nahi, MD, PhD, Jie Tang, Peter Hellemans, Brenda Tromp, MSc, Pamela L. Clemens, PhD, Andrew Farnsworth, Jesus F. San-Miguel, MD, PhD, and Saad Z. Usmani, MD
\end{abstract}

Visit http://www.bloodjournal.org/content/132/ Suppl_1/1995 for a complete list of contributor affiliations and full graphics.

Introduction: Daratumumab (DARA) is a human IgGK anti-CD38 monoclonal antibody with a direct on-tumor and immunomodulatory mechanism of action. DARA (16 mg/ $\mathrm{kg}$ administered intravenously [IV]) is approved in many countries as monotherapy and in combination with standard of care (SOC) treatment regimens for patients with relapsed/refractory (RR) multiple myeloma (MM) and newly diagnosed MM. Three phase 3 studies have now demonstrated that DARA in combination with SOC treatment doubles complete response (CR) rates, triples minimal residual disease-negative rates, and reduces the risk of progression or death by at least $50 \%$ vs SOC alone. The median durations of the first, second, and subsequent DARA IV infusions are 7.0, 4.3, and 3.4 hours, respectively. To determine whether the duration of infusion can be shortened without compromising the safety or efficacy of daratumumab, an open-label, multicenter, phase $1 \mathrm{~b}$ clinical trial (PAVO; NCT02519452) was conducted to evaluate a subcutaneous (SC) formulation of DARA with recombinant human hyaluronidase enzyme $\mathrm{PH} 20$ (rHuPH20; ENHANZE ${ }^{\circledR}$ drug delivery technol-

J Adv Pract Oncol 2019;10(suppl 2):4-9

https://doi.org/10.6004/jadpro.2019.10.2.16 ogy, Halozyme, Inc.) in patients with RRMM. Part 1 of the study revealed that a mix-and-deliver SC administration was well tolerated, with low rates of infusion-related reactions (IRRs) and similar efficacy to DARA IV (Usmani et al. ASH 2016; abstract 1149). Here, we present updated safety and efficacy findings from Part 2 of the PAVO study, where a concentrated, pre-mixed SC co-formulation of DARA and rHuPH20 (DARA SC) was evaluated in patients with RRMM.

Methods: Eligible RRMM patients had received $\geq 2$ prior lines of therapy, including a proteasome inhibitor (PI) and immunomodulatory drug (IMiD). In Part 2 of the study, a concentrated co-formulation of DARA $(1,800 \mathrm{mg})$ and rHuPH20 (30,000 U; in $15 \mathrm{~mL})$ in a single, premixed vial was administered over 3 to 5 minutes by manual SC injection into the abdomen. DARA SC was given QW during Cycles 1-2, Q2W during Cycles 3-6, and Q4W thereafter in 28-day cycles. Pre- and/or post infusion medications included acetaminophen, diphenhydramine, montelukast, and methylprednisolone. Co-primary endpoints were safety and $\mathrm{C}_{\text {trough }}$ of DARA SC at the end of QW dosing. Secondary endpoints included the overall response rate (ORR) and CR rate according to the International Myeloma Working Group response criteria.

Results: At the clinical cut-off date of December 13, 2017, 25 patients were enrolled in Part 2 of the study. Patients received a median of 3 (range: 2-9) prior lines of therapy, with $56 \%$ double refractory to both PI and IMiD. No treatment discontinuations occurred due to treatment-emergent adverse events (TEAEs). The most common ( $\geq 20 \%)$ TEAEs included lymphopenia (32\%), thrombocytopenia (24\%), fatigue, asthenia, back pain, diarrhea, nausea, headache, and viral upper respiratory tract infection (20\% each). The incidence (16\%) and severity of IRRs (mostly grade 1-2) with DARA SC was low, the majority of which occurred on Cycle 1 Day 1 , and no discontinuations due to IRRs were observed. Transient grade 3 hypertension was report- 
ed as an IRR in 2 patients. Grade 1 injection-site TEAEs were reported with DARA SC in 3 patients (induration, erythema, injection-site discoloration, and hematoma $[\mathrm{n}=1$ each $]$ ).

At 6.5 months of median follow-up, the ORR was $52 \%$ (28\% very good partial response; $24 \%$ partial response). Median progression-free survival (PFS) was not reached among all-treated patients, including among patients who were double refractory. Updated data will be presented at the meeting based on longer follow-up.

\section{The Advanced Practitioner Perspective: Josh Epworth, ARNP}

Daratumumab has proven to be a highly effective medication in treating multiple myeloma. When originally tested as a monotherapy in heavily pretreated patients, it showed enormous promise. In later studies, daratumumab plus standard of care therapy exhibited a significant improvement in the rate of complete response and increased the occurrence of minimal residual disease (MRD) negativity. Some drawbacks with daratumumab IV are that it has a relatively high level of infusion-related reactions (IRRs) in the initial doses and lengthy infusion times of between 4 and 8 hours. There has been a study that tested a 90-minute infusion of daratumumab, which has allowed for shorter infusions at some centers.

\section{The PAVo Trial}

The goal of this multicenter, open-label phase Ib trial was to determine if daratumumab could be given in a subcutaneous (SC) infusion over 3 to 5 minutes into the abdomen without compromising safety or efficacy.

The frequency of subcutaneous infusions was similar to the schedule of IV daratumumab. The scheduling and dosing of pre- and post-infusion medications was the same as well, and included acetaminophen, diphenhydramine, montelukast, and steroids.
Conclusions: DARA SC enabled dosing in 3-5 minutes and improves patient convenience. DARA SC was well-tolerated in patients with RRMM with low rates of IRRs and no new safety signals compared with DARA IV. Over $50 \%$ of patients responded to treatment and median PFS has not been reached after median follow-up of 6.5 months. These data inform ongoing phase 3 studies of DARA SC in RRMM (including a non-inferiority study of DARA SC vs DARA IV [COLUMBA; NCT03277105]), smoldering multiple myeloma, and $\mathrm{AL}$ amyloidosis.

The results of this study were promising. Regarding IRRs, the overall incidence was $16 \%$, and the severity was mostly grade $1 / 2$ and typically occurred on cycle 1 day 1 of therapy, as is typical for daratumumab IV. These reactions included hypertension and chills, allergenic rhinitis, and sneezing. Treatment-emergent adverse events (TEAEs) included lymphopenia, thrombocytopenia, fatigue, back pain, viral upper respiratory infections, nausea, and diarrhea. Injection site reactions occurred and included discoloration, erythema, induration, and hematoma.

\section{Efficacy}

Regarding efficacy, at a 6.5-month follow-up the overall response rate was $52 \%$, and median progression-free survival had not been reached.

In this 25-patient study, SC daratumumab exhibited efficacy against the disease, it was well tolerated, and patients spent significantly less time in infusion. IRRs and TEAEs were similar to those found in IV infusions of this medication, with the exception of injection site reactions. Going forward, SC daratumumab will be compared to IV daratumumab in a phase III non-inferiority study. If the SC route for daratumumab is found to be non-inferior to the IV route, this could result in the combination of the significant improvement in disease control, with much less time spent in infusion centers. 


\section{Abstract 301}

Maintenance Therapy With the Oral Proteasome Inhibitor (PI) Ixazomib Significantly Prolongs Progression-Free Survival (PFS) Following Autologous Stem Cell Transplantation (ASCT) in Patients With Newly Diagnosed Multiple Myeloma (NDMM): Phase 3 TOURMALINE-MM3 Trial

Meletios A Dimopoulos, MD, Francesca Gay, MD, PhD, Fredrik H. Schjesvold, MD PhD, Meral Beksac, MD, Roman Hajek, Katja Weisel, MD*, Hartmut Goldschmidt, MD, Vladimir Maisnar, MD, Philippe Moreau, Chang-Ki Min, MD, Agnieszka Pluta, MD, Wee Joo Chng, MB ChB, PhD, FRCP(UK), FRCPath, FAMS, Martin Kaiser, MD, Sonja Zweegman, MD, PhD, Maria-Victoria Mateos, MD, PhD, Andrew Spencer, MBBS, FRACP, FRCPA, DM, Shinsuke lida, MD, PhD, Gareth Morgan, MD, Zhaoyang Teng, PhD, Kaveri Suryanarayan, MD, Tomas Skacel, MD, PhD, Ajeeta B. Dash, PhD, Richard Labotka, MD, and S. Vincent Rajkumar, MD

Visit http://www.bloodjournal.org/content/132/ Suppl_1/301 for a complete list of contributor affiliations and full graphics.

Introduction: Maintenance therapy has been extensively explored as a strategy for prolonging the duration of disease control and potentially survival following ASCT. To date, only lenalidomide has been approved for this indication. However, lenalidomide maintenance is associated with the development of second primary malignancies and tolerability issues. PIs are a backbone of MM treatment, and bortezomib-based maintenance has shown promising activity post-ASCT, yet the benefit of PIbased maintenance has not been demonstrated in a phase 3 trial vs placebo. Moreover, the feasibility of bortezomib maintenance in routine clinical practice is limited due to tolerability and the need for regular parenteral administration. There is a need for an oral PI maintenance therapy that can be administered for a prolonged period, improve depth of response without cumulative or late-onset toxicity, and improve convenience for patients.

Methods: The phase 3, double-blind, placebo-controlled, multicenter TOURMALINE-MM3 study (NCT02181413) compared weekly ixazomib vs placebo maintenance in NDMM patients who had at least a partial response ( $\geq \mathrm{PR}$ ) to induction therapy with a PI and/or immunomodulatory drug (IMiD) followed by single ASCT. Patients were randomized $(3: 2)$ to receive ixazomib or matched placebo on days 1,8 , and 15 of 28-day cycles for up to 2 years or until progressive disease (PD) or unacceptable toxicity. Randomization was stratified by induction regimen (PI without IMiD vs IMiD without PI vs PI+IMiD), pre-induction ISS stage (I vs II or III), and post-ASCT response (complete response [CR] or very good partial response [VGPR] vs $\mathrm{PR}$ ). Patients were ineligible if they had received post-ASCT consolidation or tandem ASCT. The ixazomib dose was $3.0 \mathrm{mg}$ during cycles $1-4$, increasing to $4 \mathrm{mg}$ from cycle 5 if tolerated during cycles $1-4$. The primary endpoint was PFS per independent review committee (IRC), who were blinded to treatment assignment. The key secondary endpoint was OS. Here, we report data from the final analysis for PFS (data cut-off: April 16, 2018).

Results: 656 patients were randomized (395 ixazomib; 261 placebo). Patient demographics were balanced between groups; overall median age was 57 years (range, $24-73$ ), $37 \%$ vs $63 \%$ had ISS I vs II or III, $59 \% / 11 \% / 30 \%$ had received PI without IMiD / IMiD without PI / PI+IMiD induction therapy, $34 \% / 45 \% / 21 \%$ had achieved CR / VGPR / PR following induction/ASCT, and 18\% had high-risk cytogenetics [del(17p), t(4;14), or $\mathrm{t}(14 ; 16)]$. After a median follow-up of 31 months with $54 \%$ of PFS events, there was a $28 \%$ reduction in the risk of progression/death, corresponding to a $39 \%$ improvement in PFS with ixazomib vs placebo (median 26.5 vs 21.3 months; hazard ratio [HR] 0.72; 95\% CI: 0.582, 0.890; p=0.002; Figure). In a landmark analysis from ASCT, PFS was 30.7 vs 24.9 months (HR 0.684; 95\% CI: 0.551, $0.848 ; \mathrm{p}<0.001)$. Median PFS2 and OS have not yet been reached in either arm. Ixazomib maintenance led to higher rates of deepened response compared with placebo (relative risk 1.41; 95\% CI: $1.10,1.80 ; \mathrm{p}=0.004)$. Conversion from documented MRD positivity at study entry to MRD negativity occurred at a higher rate with ixazomib compared with placebo (12\% vs $7 \%$ ). PFS benefit was seen broadly across subgroups, including ISS III (HR 0.661), PI-exposed (HR 0.750), PI-naïve (HR 0.497 ), and patients with high-risk cytogenetics (HR 0.625). Discontinuation due to AEs was low ( $7 \%$ ixazomib vs $5 \%$ placebo). With ixazomib vs placebo, $42 \%$ vs $26 \%$ of patients had grade $\geq 3 \mathrm{AEs}$; $27 \%$ vs $20 \%$ had serious AEs; and 1 patient vs 0 died on treatment. Common grade $\geq 3$ AEs were infections ( $15 \%$ vs $8 \%$ ) including pneumonia ( $6 \%$ 


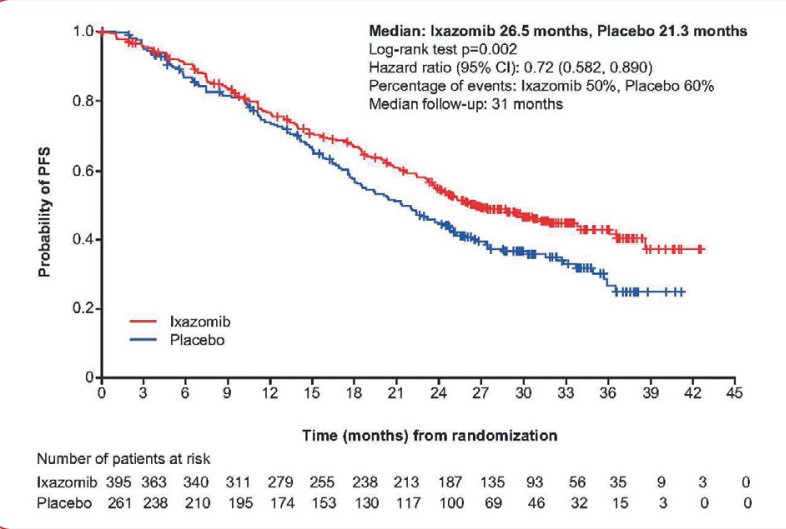

Figure 1. Probability of progression-free survival.

vs $4 \%$ ), gastrointestinal disorders ( $6 \%$ vs $1 \%$ ), neutropenia ( $5 \%$ vs $3 \%$ ), and thrombocytopenia ( $5 \%$

The Advanced Practitioner Perspective: Josh Epworth, ARNP

Maintenance therapy is a critical element in prolonging the duration of disease control with multiple myeloma following therapeutic chemotherapy with or without an auto stem cell transplant. Lenalidomide is currently the only approved maintenance medication for multiple myeloma. While effective, patients who take medications like lenalidomide and pomalidomide as maintenance can have difficulties with long-term tolerability as well as the risk of secondary malignancies. Proteasome inhibitors (PI), such as bortezomib and carfilzomib, have shown potential for maintaining control of the disease, but they are parenteral medications and as such, require regular visits to an infusion center.

\section{TOURMALINE-MM3}

TOURMALINE-MM3 is a multicenter, phase III double-blind, placebo-controlled study comparing an oral proteasome inhibitor, ixazomib vs. placebo maintenance in newly diagnosed multiple myeloma patients who achieved at least a partial response with treatment including a proteasome inhibitor and/or immunomodulator followed by an auto stem cell transplant.

656 patients were randomized (395 ixazomib, 261 placebo). At a median 31-month follow-up, median progression-free survival (PFS) with ixazomib vs. placebo was 26.5 vs. 21.3 months. Conversion of minimal residual vs $<1 \%)$. Peripheral neuropathy rates were $19 \%$ vs $15 \%$ ( $<1 \%$ vs 0 grade 3 ). Rate of second primary malignancies was 3\% in both arms. Global Quality of Life scores (EORTC QLQ-C30) on ixazomib were similar to placebo.

Conclusions: This study demonstrated a $28 \%$ reduction in the risk of progression/death, corresponding to a $39 \%$ improvement in PFS with ixazomib maintenance, with deepening of responses and increased conversions to MRD negativity over control, as well as a favorable safety profile, including an absence of risk of second primary malignancies and low rates of peripheral neuropathy, supporting ixazomib as a valuable option for maintenance therapy in responding patients post-ASCT.

disease from positive to negative occurred at a higher rate in the ixazomib group than the placebo group. Adverse events requiring discontinuation of ixazomib vs. placebo were low, with common issues including infections, gastrointestinal disorders, neutropenia, thrombocytopenia, and peripheral neuropathy. The occurrence of secondary malignancy was 3\% in each arm of the study, and quality-of-life scores were similar between the arms. As with all PIs, varicella zoster virus prophylaxis with either valacyclovir or acyclovir is required.

\section{Maintenance Option}

This study was developed to determine if ixazomib had value as a maintenance medication for people with multiple myeloma. In unrelated studies, both parenteral-dosed PIs and oral dosing of immunomodulators exhibited greater durations of PFS than ixazomib. Ixazomib appears to give an approximate 5-month increase in PFS compared to placebo.

When making the decision on maintenance therapy, clinicians should consider whether concern over secondary malignancy is significant and/or access or practicality of frequent infusions is not an option. If these issues are present, ixazomib can give patients an option for maintenance therapy with a reduced risk of secondary malignancies that allows for the administration of a proteasome inhibitor and the convenience of an oral agent. 


\section{Abstract 598}

Results of the Pivotal STORM Study (Part 2) in Penta-Refractory Multiple Myeloma (MM): Deep and Durable Responses With Oral Selinexor Plus Low Dose Dexamethasone in Patients With Penta-Refractory MM

Ajai Chari, MD, Dan T. Vogl, MD, Meletios A Dimopoulos, MD, Ajay K Nooka, MD, MPH, Carol Ann Huff, MD, Philippe Moreau, Craig E. Cole, MD, Joshua Richter, David Dingli, MD, PhD, Ravi Vij, Sascha A. Tuchman, MD, Marc S. Raab, MD, PhD, Katja Weisel, MD, Michel Delforge, MD PhD, David Kaminetzky, MD, Robert Frank Cornell, MD, A. Keith Stewart, James Hoffman, Kelly $N$. Godby, MD, Terri L. Parker, MD, Moshe Levy, MD, Martin Schreder, MD, Nathalie Meuleman, PhD, MD, Laurent Frenzel, MD, PhD, Mohamad Mohty, MD, PhD, Choquet Sylvain, MD, Andrew J. Yee, MD, Maria Gavriatopoulou, MD, Luciano J. Costa, MD, PhD, Jatin J. Shah, MD, Carla Picklesimer, Jean-Richard Saint-Martin, Lingling Li, Michael G. Kauffman, MD, PhD, Sharon Shacham, PhD, $M B A$, Paul Richardson, and Sundar Jagannath, MD

Visit http://www.bloodjournal.org/content/132/ Suppl_1/598 for a complete list of contributor affiliations and full graphics.

Introduction: Selinexor is a novel, oral Selective Inhibitor of Nuclear Export (SINE) compound that

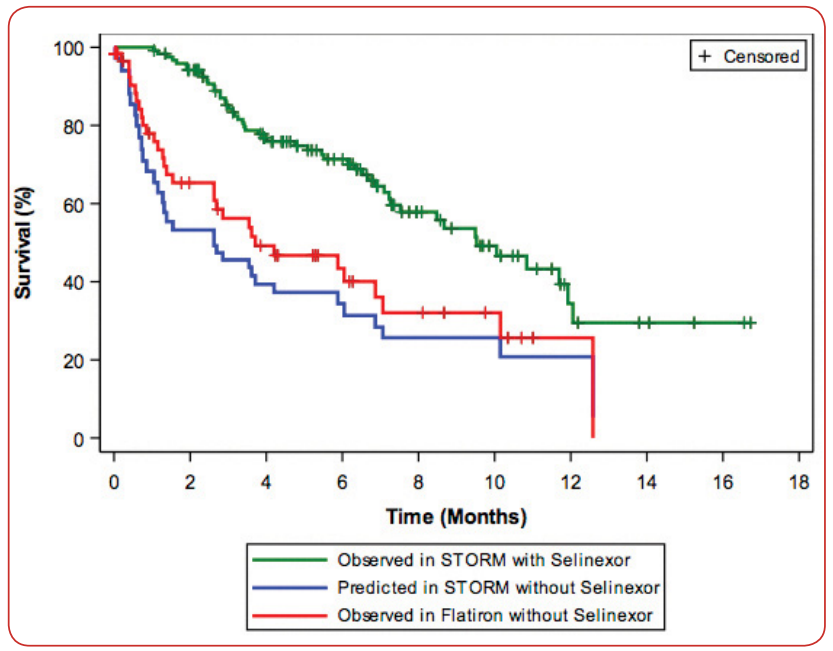

Figure 1. Median Overall Survival using Predictive Modelling Approach by Study Population Database Cutoff Dates: 2018-03-31 (FHAD) and 2018-05-17 (STORM) FHAD: Flatiron Health AnaIytic Database.

Primary efficacy population consists of patients in FHAD with documented triple class refractory multiple myeloma with baseline ECOG performance status of $0,1,2$, or missing and patients in the mITT population of the STORM study. ${ }^{2} \mathrm{~K}-\mathrm{M}$ curve for Predicted in STORM without selinexor (blue) was estimated by applying parameter estimates observed for prognostic factors of OS in Flatiron to the STORM study population. blocks exportin 1 (XPO1). Selinexor treatment results in nuclear accumulation and activation of tumor suppressor proteins, inhibition of NF-kB, and translational suppression of several oncoprotein mRNAs (e.g., c-myc, cyclin D). Multiple myeloma (MM) remains incurable, and most patients (pts) eventually progress through standard drug classes of proteasome inhibitors (PIs), immunomodulatory drugs (IMiDs), anti-CD38 mAbs and others. The increased use of combinations in MM treatment, (PIs/IMiDs/mAbs), has led to a growing number of pts with penta-refractory MM (pts that have been treated with bortezomib (bort), carfilzomib (carfil), lenalidomide (len), pomalidomide (pom) and daratumumab (dara)). Active novel therapies with different mechanisms of actions are needed to address this unmet medical need. Part 1 of STORM enrolled pts with both quad- (bort, carfil, len, pom, treated MM) or penta-refractory MM and demonstrated an overall response rate (ORR) of $21 \%$ (Vogl et al, JCO 2018). Based on these findings, the Pivotal Part 2 of STORM was initiated, enrolling an additional cohort of 122 patients with penta-refractory MM.

Methods: Pts with penta-refractory MM were treated with $80 \mathrm{mg}$ selinexor plus $20 \mathrm{mg}$ dexamethasone (Sd) twice weekly. Pts must have received an alkylator, bort, carfil, len, pom and dara, and had MM refractory to $\geq 1 \mathrm{PI}, \geq 1 \mathrm{IMiD}$, dara, a glucocorticoid, and their last therapy. Pts must have a total ANC $\geq 1000 \mathrm{~mm}^{3}$, platelets $\geq 50 \mathrm{k} / \mathrm{mm}^{3}$ (or $\geq 75 \mathrm{k}$ if marrow plasma cells $<50 \%$ ), and creatinine clearance $\geq 20 \mathrm{~mL} / \mathrm{min}$. The primary endpoint was ORR. Secondary endpoints: duration of response (DOR), clinical benefit rate (CBR), progression free survival (PFS), and overall survival (OS). Efficacy was assessed by an Independent Review Committee (IRC) based on IMWG criteria. OS was also compared to a cohort of pts with PI, IMiD, dara refractory MM from the Flatiron Health Analytic Database (FHAD), (ref: ASH 2018 abs ID: 116493), who met all the inclusion criteria for STORM.

Results: As of 1-Jun-2018, 122 pts (71 M/ $51 \mathrm{~F})$ were enrolled in 38 sites (US and EU). Pt characteristics were [medians (range)]: age 65 yrs (40-85); 7 (3 18) prior treatment regimens, $6.6 \mathrm{yrs}(<1-23.4)$ from initial MM diagnosis.65 pts (53\%) had high risk cytogenetics, 86 pts (70\%) had prior dara in combination, 102 pts (84\%) had prior stem cell transplantation, 2 pts had prior CAR-T therapy. All pts enrolled with 
progressive disease (PD), 72\% of pts had increases (3\% - 792\%) in MM markers from screening to C1D1 (median 11 days). Frequently reported Sd treatment related adverse events (AEs) included (all grades, grades 3/4): thrombocytopenia $(67 \%, 53 \%)$, nausea $(67 \%, 10 \%)$, fatigue $(68 \%, 21 \%)$, anorexia $(50 \%$, $2 \%)$, anemia $(46 \%, 28 \%)$, and weight loss (46\%, $0 \%)$. Eight pts remain on study and 114 pts discontinued treatment (most commonly for PD). There were 4 deaths on treatment: sepsis, respiratory failure, pulmonary embolism, and an unrelated, unspecified cardiac event. IRC determined ORR ( $\geq \mathrm{PR})$ was $26.2 \%$, with $6.5 \% \geq$ very good partial response, including 2 stringent complete responses (sCRs; MRD negative at 1:10-6 and at 1:10-4sensitivity). Both pts who relapsed after CAR-T achieved PRs. The CBR ( $\geq$ minimal response, $[\mathrm{MR}]$ ) was $39.3 \%$, and $79 \%$ of pts achieved $\geq$ stable disease (SD). Responses typically occurred within the first month. Medians: DOR 4.4 months (mo) (range $<1-10 \mathrm{mo}$ ), PFS $3.7 \mathrm{mo}$, and

\section{The Advanced Practitioner Perspective: Josh Epworth, ARNP}

Selinexor is an oral, first-in-class selective inhibitor of nuclear export that specifically blocks nuclear export protein (XPO1) from shifting tumor suppressor (TSP) proteins from the nucleus of cells. In the MM setting, XPO1 is overexpressed, resulting in elevated transport of TSP from the nucleus, allowing for decreased resistance to disease progression. Selinexor works by blocking XPO1, allowing for an accumulation of TSPs in the nucleus that is believed to result in a suppression of cancer cells while sparing normal cells.

\section{The STORM 2 Trial}

STORM 2, a multicenter, phase II clinical trial, evaluated selinexor in patients with MM refractory to bortezomib, carfilzomib, lenalidomide, and pomalidomide (quad), with a subset also refractory to an anti-CD38 antibody (penta). 122 patients were enrolled, 8 remained on study, and the most common reason for discontinuation was progression of disease. ORR greater than or equal to PR was $26.2 \%$, with most responses occurring within the first month. The PFS was 3.7 months, and OS was 8 months.

In this study, side effects, including grade 3/4 adverse events, included thrombocytopenia and anemia as well as hyponatremia, nausea, and fatigue. The abstract notes that these side
OS 8.0 mo. Pts with $\geq$ MR had significantly longer OS than pts with $\mathrm{PD} / \mathrm{NE}$ (median not reached vs $1.9 \mathrm{mo}$, $\mathrm{p}=<0.0001)$. Compared to the FHAD cohort, STORM cohort had longer OS (Figure 1, HR 0.41, $\mathrm{p}=0.0001$ ).

Conclusions: Results of the pivotal STORM Part 2 in penta (PI, IMiD, dara)-refractory MM demonstrated that oral selinexor plus low-dose dexamethasone (Sd) was highly active with an ORR of $26.2 \%$. Importantly, responses were rapid and deep with 2 patients achieving sCRs (both MRD negative) in these heavily pre-treated penta-refractory MM pts (median 7 prior regimens, 53\% high risk). AEs are a function of dose/schedule/disease severity and can be managed with dose modifications and supportive care. No major organ toxicity was observed and AEs were typically transient and reversible. Sd is an all-oral, first in class mechanism with novel MOA and represents a potential therapeutic option to the growing number of pts with penta-refractory MM who have exhausted approved therapies.

effects were managed by dose modifications and supportive care. When using this medication, the use of prophylactic anti-emetics and close monitoring of counts is advised.

Selinexor has a novel mechanism of action that exhibited effect against the disease as a monotherapy in heavily pretreated patients. In the STORM 2 study, a significant number of patients did not complete the trial because of progression of disease, and the duration of PFS was limited to less than 4 months.

\section{Potential Future Directions}

Selinexor is not currently FDA approved for the treatment of MM, yet it has been granted priority review for MM. A number of studies are ongoing for its use in combination with standard of care. Examples include BOSTON, a study comparing bortezomib + dexamethasone vs. selinexor + bortezomib + dexamethasone, and STOMP, which combines selinexor with lenalidomide and dexamethasone. Recently, the Oncologic Drug Advisory Committee (ODAC) decided against recommending accelerated approval of selinexor for the treatment of patients with relapsed/refractory MM, citing difficulty determining whether the benefits outweighed the risks. The ODAC has advised that the FDA await results from BOSTON before deciding on approval. Results from BOSTON are expected in 2020. 